Full citation: Clear, T., \& MacDonell, S.G. (2010) Beyond 'Temponomics' - the many dimensions of time in globally distributed project teams, in Proceedings of the 2010 International Conference on Global Software Engineering (ICGSE 2010). Princeton NJ, USA, IEEE Computer Society Press, pp.297-304.

doi: 10.1109/ICGSE.2010.56

\title{
Beyond 'Temponomics' - The Many Dimensions of Time in Globally Distributed Project Teams
}

\author{
Tony Clear and Stephen MacDonell \\ SERL, School of Computing \& Mathematical Sciences \\ Auckland University of Technology \\ Private Bag 92006, Auckland 1142, New Zealand \\ tony.clear@aut.ac.nz,stephen.macdonell@aut.ac.nz
}

\begin{abstract}
The prevailing notion of time which pervades reports on global software development practice is the linear notion of time as a scarce commodity to be optimized through working across global boundaries. This 'temponomic' view of time provides a useful but limited model through which to understand how time operates in practice within globally distributed teams. We report findings from an in depth empirical study which employed a grounded analysis of the many dimensions of time in action within a global team setting. A situated analysis of the actions at each of three globally distributed sites, demonstrates how the differing aspects of time interact, and how some of the known challenges in working globally, can be viewed from a temporal viewpoint. We argue that this more nuanced understanding of how time functions in globally distributed teams may help managers and researchers develop more appropriate practices and models for managing such teams.
\end{abstract}

Keywords: time, globally distributed teams, global software development

\section{INTRODUCTION}

While some investigations into the phenomenon of time and its operation within globally distributed teams have been undertaken, the prevailing notion of time apparent in the literature and which pervades reported global software development practice (e.g. the "follow the sun" [1] and tightly structured three location " 24 hour factory" models outlined in [2, 3]) is the linear notion of time as a scarce commodity to be optimized through working across global boundaries.

This 'temponomic' [4, p.61] view of time provides a useful but very limited model through which to understand how time operates in practice within globally distributed teams. The virtual context provides a fertile field for research as observed in [5], who call for further research "to limit the negative effects of time dispersion".

This paper reports findings from an in depth empirical study which has employed a grounded analysis of the many dimensions of time in action within a global team setting. This analysis of a rich body of data extends prior work that has promoted the multi-dimensional nature of time, by demonstrating the operation of time at the micro level within a distributed educational team setting. Viewing the structuring of time from several perspectives, the complex, subtle and frequently conflicting elements of time are shown to combine to create a dynamic that the commercial view that 'time is money' fails to adequately recognize.

We first present a brief perspective on the concept of 'time' in the context of global teams and global software development, followed by an overview of the study reported here. Then follows a situated analysis of relevant actions at each of three globally distributed sites, through a set of selected episodes. In demonstrating how the differing aspects of time interact, the paper explains how some of the known challenges in working globally, such as planning around holidays and festivals [1], can be viewed not solely from a cultural perspective but from a temporal viewpoint.

It is argued that a more nuanced understanding of how time functions in globally distributed teams may help managers and researchers develop more appropriate practices and models for managing such teams. The paper concludes with recommendations for better accommodating the temporal dimension in global team contexts.

\section{THE QUESTION OF TIME}

Time is a concept that we frequently take for granted, but as noted in the intriguing analysis presented in [4] it is a 
multi-faceted, complex, culturally bound and socially constructed entity.

Time has received some attention in the literature relating to global software development (GSD) [6, 7, 2, 3, 1], but has been more extensively addressed in the literature relating to groups and their development $[8,9,10,11,12$, 13] and as an extension to that within the literature relating to global virtual teams [14, 15, 16, 17]. Broader discussions of time have also been presented from the point of view of research design $[18,19]$, of culture [20, 21 , and of time in operation in organizational or wider contexts [22, 23, 4, 24, 25].

Unpacking this diverse body of literature presents many differing perspectives on time, including clock time, time as duration, the need to synchronize time across global systems and time zones, and varying cultural views of time (e.g. long term versus short term orientation [21] or sequential versus synchronous views of time [20]). Here a relevant distinction is whether time is viewed as monochronic: "When time is considered tangible, it is conceived as a commodity... Monochronic individuals tend to view time as a tangible resource (i.e., something to be saved or wasted)" [24]; or polychronic "When time is considered intangible, activities are typically event-based. Time is viewed as a 'backdrop against which events unfold'...polychronic individuals tend to view time as an intangible resource that is not directly controllable. Activities are timed by events rather than a clock" [24]. The notion of managerial challenges inherent in balancing 'scheduled time' versus 'kairotic time' is also presented in [25, p.22] who argue that "the traditional factory model of organizing should give way to dispersed calculation and kairotic time (where kairotic time is understood to be temporal autonomy, such that work gets done at the right time, rather than when mandated on a calendar or project plan)”.

This necessarily brief review of the concept of time merely aims to illustrate its innate complexity and the futility of producing any simple model or framework for analysing and predicting models of time in operation within a GSD context. We will return to the question of a suitable framework later after first discussing our field study and the dimensions of time which became evident as a result of the empirical analysis. But as one comment here it can readily be seen that "the 24 hour factory" model of [2] and [3] is based upon a monochronic perspective on time and in direct opposition to alternative perspectives.

\section{STUDY CONTEXT}

The field study reported here investigated the actions of participants in a three site global collaboration trial (AUT University, New Zealand; St Louis University, Missouri; Uppsala University, Sweden), carried out in late 2004. The collaborative process was carried out with the support of a Web based environment, consisting of two primary components: the virtual learning environment at AUT "AUTOnline" based upon the Blackboard ${ }^{\mathrm{TM}}$ commercial platform; and a collaborative database custom built in Lotus Notes. The Web based environment was designed, set up and administered at AUT University in New Zealand.

Students were assigned to Global Virtual Teams (GVTs) and required to participate in a collaborative process that consisted of two phases: Icebreaking and Group decisionmaking. Each GVT had access to their own asynchronous (discussion forums) and synchronous (chat) facilities, and to file-sharing and individual homepage features. They also had access to a collaborative database VTeam that was custom-built to support and record their team decision making process and their evaluations of the exercise.

The Icebreaking phase was designed to allow GVT members to get to know each other and to establish their group dynamics. The GVTs were given a choice of communication means as well as the options of either choosing a team leader or deciding on a self-managed team. The second phase of the collaboration involved group decision making related to a common task. Each GVT was expected to identify, upload and evaluate a selection of Web sites related to collaborative technologies. They were required to discuss the web sites based on a suggested evaluation framework and to reach group consensus on the final ranking of the sites. The second phase was to be carried out in the custom built collaborative environment. The two collaboration phases were planned for the period between 6th and 19th September and (due to an intervening two week semester break at the Auckland site) from the 4th October to 1st November 2004.

During the process of preparing for the trial, three main tiers emerged in the overall structure: a) students organised in Global Virtual Teams; b) faculty members in their multiple roles of lecturers, coordinators and facilitators; and c) institutional infrastructure (including staffing) for both IT and logistical support.

The students at each location were further divided into small local teams (LTs). Each LT was part of a Global Virtual Team (GVT) along with one or two LTs from other locations. There were 9 GVTs altogether that ranged in size between 5 and 9 members, as the differing number of students at each site meant that some teams could draw from two sites only.

At each of the three locations the collaboration was part of a course assessment and there were lecturers who coordinated the collaborative process locally. At the New Zealand location one of the lecturers was also a trial facilitator who had to ensure that all local coordinators at each of the three participating sites were provided with correct and timely information about the trial setup and any administrative issues.

The Web-based platform for the collaboration was set up and administered by the Flexible Learning Services and Information Technology (IT) Support groups at AUT and all communication with them was maintained by the trial facilitator. There was also some IT support provided locally at the Uppsala and University of St Louis sites.

While this was an educational collaboration during which students from all three sites worked collaboratively to achieve a common goal, the focus of the study was more 
specifically on those involved in coordinating and supporting the global collaboration, and in particular their roles and activities of "technology-use mediation" [26, 27]. Namely, how they established the technology for the collaboration, how they reinforced and adjusted patterns of use and how they periodically undertook considered major revisions of the supporting technology platforms. Thus while software designer and developer may have been inherent roles for those establishing the technology platform (a custom Lotus Notes application reinforced by the standard AUT University virtual learning environment), the context was more one of a global virtual team of educational and IT professionals than of global software developers.

That said, the issues encountered here are applicable to a wide range of global virtual teams. They are very much relevant to distributed software teams, where equivalents to many of the 37 independently coded roles identified in this project (e.g. coordinators, team leaders, system support consultants, testers, configurers, trainers and offshore technical co-ordinators [28]) can be found.

The data for the study consisted primarily of a large corpus of email messages spanning more than a year's duration, and covering the phases of the collaboration from inception to completion. This material was complemented by a set of extensive research diary notes, online postings and questionnaire responses, and various documentary artefacts such as: course outlines; instructions to participants; human subject ethics approval documents and assessment guidelines. This presented a large body of textual and digital information for analysis.

\section{EMPIRICAL DATA ANALYSIS}

The analysis first addressed the email data, which comprised a set of what has been termed "mosaic messages" [29] with embedded responses and response to responses. Initial scrubbing and cleansing of the email messages, involved breaking down the original 175 messages into their component parts resulting in some 1086 segments which after scrubbing and removing duplicates resulted in 332 unique message segments for analysis.

The analysis proceeded through a grounded theoretic investigation based upon a "theoretical sampling" strategy involving selection of specific episodes deemed representative of the four phases of technology-use mediation (establishment, reinforcement, adjustment, and episodic change). An episode of interest was defined as:

A relevant temporally bound sequence of events with antecedent conditions and outcomes, which stands apart from others, and has been selected for analysis. [27, p. 62)

This sampling approach was consistent with the recommendations on grounded theory presented in [30, p. 62-63].

"It is important to contrast theoretical sampling based on the saturation of categories, with statistical (random sampling). Theoretical sampling is done in order to discover categories and their properties, and to suggest the interrelationships into a theory...The adequate theoretical sample is judged on the basis of how widely and diversely the analyst chose his groups for saturating categories according to the type of theory he wished to develop".

The data within each of the selected episodes thus presented a more manageable subset of the overall corpus. This data augmented by relevant diary notes and electronic or documentary artefacts for the time window in question, was then subjected to a process of detailed coding based upon reading each excerpt and drawing forth discrete codes from the text. Recurring codes were then collated into broader concepts drawn directly from the data comprising the episode.

Eight episodes deemed to be broadly representative were selected for the study, and codes and concepts were progressively derived by detailed analysis of each episode. This set of eight episodes comprised: one lengthy episode based on a large body of email data covering the initial establishment phase of the project; four episodes covering adjustment/reinforcement TUM activity modes; and three (based mostly on critical incidents) addressing the episodic change mode.

Time was but one element of many in the rich collection of codes and concepts that resulted from the analysis. For Giddens "one of the dominant characteristics of modernity is the separation of time from space made possible by the standardization of time across the world", with "human efforts to standardize temporal frameworks inscribed in official time zones" [22]. Therefore time is addressed here as a topic of particular interest in global virtual teams and in the GSD context. However it must be acknowledged that time and space logically belong together in a fuller analysis, but space limitations here demand a focus on time alone as a rich enough concept in its own right. Cross episode comparisons demonstrated that some consistent patterns of concepts became apparent and the treatment of time had some commonality across episodes.

The data was subjected to an additional form of analysis by concentrating upon the dynamic structuring of time, through a structurational analysis. Such analysis aims to integrate the three sensitizing devices for Structuration Theory research recommended in [31] within each episode, namely illuminating: the operation of duality of structure; the role of time/space and revealing the actors' knowledgeability or "reflexivity regarding their day-today interactions” [31, p. 1367). Space precludes a fuller exposition of Structuration Theory in this paper, and the reader is referred to [31] for further elaboration. For the purposes of this paper a structurational analysis is a form of interpretive analysis which demonstrates the 'structuring' of a set of social dynamics, or the ways in which patterns of activity are initiated, become established and are sustained in a given context. The specific aspect given additional attention in this paper is the dynamic process through which the operation of time is portrayed within the selected episodes. Thus the dynamic structural analysis serves to complement the coding and concept identification process of grounded theory. 


\section{FINDINGS}

In this section we first tabulate the time related codes and concepts derived from selected individual episodes of technology-use mediation activity, demonstrating some common patterns across the episodes selected for the study. This tabulation is then augmented by mapping the concepts to a broader framework for analysing time. The implications of this framing are discussed, then illuminated in more depth through a study of the data underlying the codes, and the way in which the structuring of time unfolds in a situated context of global practice.

\section{A. Adjustment /Reinforcement Episode}

This brief episode covered a two day period in the middle of the collaboration reported in two diary notes of some 432 words of text. Notes of a telephone conversation between two of the coordinators - one in New Zealand and one in Sweden - were recorded, followed by a subsequent updating discussion between two of the NZ coordinators. The episode covers the technology-use mediation activities of adjustment and reinforcement. Despite the limited amount of text analyzed, the coding produced the pattern of time related codes (based upon the actual words identified in the text and the concepts extrapolated from them) in table I below.

TABLE I. TIME RELATED CODES FROM ADJUSTMENT/ REINFORCEMENT EPISODE

\begin{tabular}{|l|l|}
\hline Concepts & Codes \\
\hline Time Pressure & busyness \\
\hline Time Pressure & concurrent tasks \\
\hline Time & Synchronize \\
\hline Time & Time separation \\
\hline Time & Time \\
\hline Time & time zone \\
\hline Time & Runestone \\
\hline Time & day \\
\hline Time & experience \\
\hline
\end{tabular}

\section{B. Episodic Change Episode}

This even briefer episode covered a meeting between the New Zealand and Swedish coordinators held at the Frontiers in Education conference in Colorado some six months prior to the collaboration. The data for this episode had been recorded in a set of meeting notes of some 765 words of text. The episode covers the technology-use mediation activities of episodic change during which a process of diagnosing some dissatisfactions with the previous year's collaboration had been conducted and a plan to address the deficiencies in the next collaboration had been drafted. The coding produced the pattern below of time related codes, which although reduced was slightly more future oriented.
TABLE II. TIME RELATED CODES FROM EPISODIC CHANGE EPISODE

\begin{tabular}{|c|c|}
\hline Concepts & Codes \\
\hline Time & stages of scripting the project \\
\hline Time & Synchronize \\
\hline Time & Time \\
\hline
\end{tabular}

\section{Establishment Episode}

This episode in contrast was far larger in duration and content, as a logical unit which covered the full set of technology-use mediation activities of establishment for the collaboration. The data for this episode traversed the period from September 2003 to October 2004, comprised some 16 different actors, 216 different data sources mostly email messages augmented with 19 associated files, and a daunting 367,973 words of text. The coding produced the richer pattern of time related codes shown in Table III. Moreover the concept of time was pervasive, being present in over $90 \%$ of the data sources analyzed. Synchronize was the most common code, found in 34\% of the 216 data sources.

TABLE III. TIME RELATED CODES FROM ESTABLISHMENT EPISODE

\begin{tabular}{|l|l|}
\hline \multicolumn{1}{|c|}{ Concepts } & \multicolumn{1}{|c|}{ Codes } \\
\hline Time Pressure & busyness \\
\hline Time & daylight-saving \\
\hline Time & Runestone \\
\hline Time & schedule \\
\hline Time & experience \\
\hline Time & stages of scripting the project \\
\hline Time & time zone \\
\hline Time & holiday \\
\hline Time & Synchronize \\
\hline Time & Time \\
\hline Time & class schedule \\
\hline Time & delay \\
\hline Time & time separation \\
\hline
\end{tabular}

\section{A Framework for Considering Time}

Arrow, Poole and colleagues in [10] refer to a classification "that identifies five types of time: clock time, cyclical time (such as the succession of seasons), event time (subdivided into predictable and unpredictable), and life cycle time that refers to development progression within a finite lifecycle span. Two examples of predictable events that structure time are paydays and holidays”. The distinction between clock and event time is also drawn in [22] and [17], with the latter classing clock "time as a scarce commodity", as opposed to event time being "cyclical, continuous (holistic), and epochal". These distinctions echo the monochronic and polychronic perspectives [24], and the 
sequential versus synchronous views of time [20] noted above. It is further noted in [10], that: "time is a fundamental (and often problematic) issue for theory and research".

Mapping the concepts and codes of table III above to the five 'types' of time of [10], gives the tabulation below (Table IV), which illustrates the multiple types of time which may simultaneously be in operation within a global collaboration. The potential for conflict between these differing types is considerable, when for example an urgent need to synchronize activities across sites encounters an unremarked change in daylight saving time, or contends with a local holiday at one site. Frequently these conflicts are overlooked, and a scheme such as the categorization below may help build a more conscious picture of the forces in contention.

TABLE IV. ESTABLISHMENT EPISODE - CODES AND TIME CLASSIFICATIONS

\begin{tabular}{|l|l|l|}
\hline \multicolumn{1}{|c|}{ Time Type } & \multicolumn{1}{c|}{ Concepts } & \multicolumn{1}{c|}{ Codes } \\
\hline Clock & Time & time zone \\
\hline Clock & Time & Time \\
\hline Clock & Time & delay \\
\hline Clock & Time & time separation \\
\hline Clock event & Time Pressure & busyness \\
\hline $\begin{array}{l}\text { Clock, cyclical, event } \\
\text { (unpredictable), lifecycle }\end{array}$ & Time & Synchronize \\
\hline $\begin{array}{l}\text { Clock, cyclical, event } \\
\text { (predictable) }\end{array}$ & Time & daylight-saving \\
\hline Clock, lifecycle & Time & schedule \\
\hline Clock, lifecycle & Time & class schedule \\
\hline $\begin{array}{l}\text { Event (predictable), } \\
\text { cyclical }\end{array}$ & Time & holiday \\
\hline Lifecycle & Time & Runestone \\
\hline Lifecycle & Time & experience \\
\hline Lifecycle & Time & stages of scripting the project \\
\hline
\end{tabular}

\section{E. The Structuring of Time}

A look beyond these abstract codes to some specific examples, may help illustrate how the differing types of time impact on activities within the global team context, and serve to structure time in practice.

Yet we must again acknowledge that addressing time independently of space (at least in this form of cross-site context) is analytically difficult. For instance synchronize as the dominant time related code from table III above, inherently involves a cross-site or 'spatial' dimension. Some cultures explicitly recognise this reality as noted in [20, p.308] where the Chinese character "for 'time' includes 'space' so that time is folded like Einsteins' universe”.

As the excerpts below indicate, Northern \& Southern hemisphere semester patterns differ, but, in order to collaborate, a common window must be found, and the course schedules and assessment components need to be synchronized:
BTW we still await confirmation of the collaborative window timings for Uppsala students. When does your course start?? (AP 17/08)

With regards to timing and numbers it could well be only about 45 people in the course this year. We need more input from $M D$ on the timing that he thinks will suit the hospital and the course layout/schedule for this year. (AP 17/08)

In this brief interchange we see the intersection of several types of time. 'Predictable event' time designated by the separate course "calendars" or schedules, and as determined both by the related schedule for the overall collaboration and the embedded Uppsala project with their academic hospital (the context for their work); implicit 'clock' time with specific dates and course session (class schedule) times to be determined; 'cyclical' time with the succession of each academic year and each annual collaboration. 'Lifecycle' time is implicit in this interchange too, occurring as it does in the 'establishment phase', in itself a stage of scripting the project.

Attempts to synchronize activities also reflect failures and delay, such as the excerpt below, arising from earlier delay and difficulties in loading students into the AUTonline system:

People here are getting a trifle frustrated with that, especially since the trial phase 1 is supposed to be concluded on the weekend. (AP 17/09)

In the above excerpt we see 'clock' time evident in the delay, with student frustration over the waste of their time 'as a resource' and the weekend as a deadline, causing time pressure. A further evident form of time is 'predictable event' time in the 'weekend' bounded schedule for 'trial phase 1', with the delay arguably shifting the schedule to 'unpredictable event' time, as also exemplified previously in this message from DK:

Lets hope that we 'll be able to start work on Tuesday. (DK 03/09)

The interchanges below evidence the five types of time of [10]: 'cyclical'; 'clock'; 'predictable event'; 'unpredictable event'; and 'lifecycle' time. The Southern hemisphere semester cycle and the Northern hemisphere holiday season, represent both recurrent therefore 'cyclical', and 'predictable event' time (by their scheduled nature) in the first communication, with the busy semester finish and start periods indicative of time pressure and 'clock' time for Southern hemisphere academics, while Northern hemisphere academics by contrast were on holiday coinciding with their 'cyclical' summer season.

We just finished the first semester, all our marking is over now and now we are on to the second semester. It starts in two weeks time and we are busy now preparing the courses. I hope you are having a good holiday but haven't forgotten about our collaborative exercise :) (DK05/07)

Please note that students from New Zealand have a two week break between the 20th of September and the 3rd of October. Members of GVTs are encouraged to carry on with the icebreaking 
process using any of the above communication channels. (DK 24/08)

Users will get to learn to meet deadlines, which is especially important when working internationally, taking in account of different time zones. (DK30/08)

'Predictable event' time and concrete 'clock' time are evident in the schedule for the semester and the courses, the two weeks until start time, the 'icebreaking phase' of the collaboration, the holiday break for New Zealand students (notably again offset from the Northern hemisphere academic schedule), and an exhortation to continue during the break to synchronize efforts within the student GVTs. The 'icebreaking phase' warrants classification against 'lifecycle' time too, reflecting its developmental role in the progression of the collaboration cycle. The third message recounts previous student feedback, which reflected upon the value of learning to synchronize work through 'predictable event' time regulated by 'deadlines' (designated as 'clock' time which itself is differentiated by time zones).

From the above discussion we see evidence of the temporal oppositions noted in [22], specifically the opposition "between universal (global, standardized, acontextual) and particular (local, situated, contextspecific) time. Discussing the manner in which "calendars, have shifted from being particularistic and local (often associated with religious communities) to being universal and global (associated with the spread of trade, industrialization and capitalism)" (ibid.), raised interesting questions for this collaboration. Is a Global Virtual Team then, inherently a product of the trend towards universal time, wherein time is the scarce resource of 'clock' time (based on what might be termed a 'temponomic' world view [1, p.61]), or in the educational context are the local and particular forces and the roots of tertiary education in religious communities, the seasons of the year and 'cyclical' time too strong?

Certainly the marrying of the Northern and Southern hemisphere seasons and academic calendars, with their uneven workload peaks, differing breaks and holidays and even differing cultural approaches to holidays, creates challenges for North South collaboration, while the three site and disparate time zone collaboration adds a further challenge in finding a window of 'clock' time that is not highly antisocial for at least one party.

As a result we have not attempted to conduct a collaboration more than once a year, as the first semester in New Zealand Universities typically begins in March, after the second semester has ended in late November. Summer school is an option, but only a very brief window is available. Then too the New Zealand cultural attitude towards holidays has traditionally meant that from late December to January (summer time), practically the whole country has 'gone to the beach'. The depth of this appreciation of an idyllic summer at the beach can be read in the iconic Katherine Mansfield short story "At the Bay” written in the 1920's [32]. 'Cyclical' time in this instance wins over global and 'clock' time for Global virtual teams.

\section{IMPLICATIONS AND RECOMMENDATIONS}

From the inherently interwoven nature of the types of time portrayed in the above section, we argue that the predominant viewpoint on time (namely 'clock time' as expressed in $[2,3,1,6])$ which presently informs global software development, is too narrow and needs review. A more nuanced understanding of how time functions in globally distributed teams may help managers and researchers develop more appropriate, mutually respectful and sustainable practices and models for managing such teams.

\section{A. Some Questions and Suggested Strategies for Managing 'Time' within Distributed Teams}

A first step for managers then is to note the significant implications of the different types of time in operation within global team settings. While managerial training may prioritise the 'time is money' and 'time as a scarce commodity' perspectives, this viewpoint is inherently constricting. It frequently overrides the value systems and cultures of local sites in favour of the global view. While this approach may prove effective short term, it is essentially dependent upon arrangements in which an unequal power balance is maintained, and some level of exploitation is inherent. We argue that it does not provide for sustainable relationships across sites and will only serve to build resentments between the parties and across sites longer term.

Having asserted the need for a wider perspective on time in distributed project settings, a useful starting point would be to consider the five types of time noted in the classification scheme of [10] and portrayed in table IV above.

When scheduling project activities some key questions and strategies for consideration are:

- What 'predictable events' need to be allowed for?

- Can these events be managed around or are they ingrained into the local culture through established 'cyclical' time patterns (e.g. holiday breaks, academic semesters, religious festivals, times of worship etc.)?

- When are periods of extreme workload and 'time pressure' likely to arise in the project? Will they contend with 'cyclical time' patterns and 'predictable events' at one or more sites? If so can work be shifted to another site, that is not so impacted?

- Have the implications of differing 'time zones' been accounted for in modifying unreasonable working hours and 'time pressure' at one or more sites? If not can locations be adjusted so that there is a common reasonably acceptable time window for handover between each site?

- Have contingency plans been put in place for managing 'delays' so that one site does not bear 
an unfair burden in its resolution, or that enables other sites to continue working on some known or agreed basis?

- Have suitable tools, techniques, practices and roles (e.g. communication technologies and documentary repositories, methodologies and project management systems, meeting scheduling and calendaring systems, version control and configuration management systems, "offshore technical co-ordinators" [28], etc.) been put in place to enable synchronization of tasks and activities across sites?

- For smaller software organisations and teams engaging in more ad-hoc collaborations, what is the minimal level of such infrastructure that may prove workable?

- At a relatively simple level, has a joint project clock and calendar been established on some form of universal time basis (cf. for instance the need for global standardization evidenced by [23]), so that changes into and out of daylight saving time can be readily accommodated across sites?

- What is the contribution of 'experience', or the duration of time over which individuals work together in forging more effective global teams?

- What development 'lifecycle' models present themselves most fruitfully in the design of distributed projects?

While the above set of questions is far from exhaustive it points to areas in which attention does need to focus, when managing distributed teams and considering the complexities of 'time' in operation within a global team context.

\section{B. Some Research Questions Relating to 'Time' within Distributed Teams}

In addition to the above questions for practice, a number of areas in which our knowledge of the functioning of time within global teams is incomplete present themselves.

We need to better understand the dominant modes of time in operation within distributed teams, and the interactions between these modes demonstrated in the everyday practices of distributed team members, by studies such as that reported here, or by engaging in longitudinal field studies of GSD teams in action. Such research may identify the most common patterns of time in operation in particular situations and contexts. Thus we may gain insight into where managing globally distributed teams and software projects under a model of 'clock time' has merit and where alternative conceptions must be considered. Determining the most appropriate balance of types of time and how to achieve balance is a related question.

The need to balance differing 'types' of time raises of course the accompanying concept of 'space' - noted in
[31] as innately inseparable from time. As phrased in [22, p.690]

"all universal temporal structures must be particularized to local contexts because they are enacted through the situated practices of specific community members in specific locations in specific time zones".

Although not addressed in this paper (other than noting that synchronization inherently has a spatial as well as a temporal dimension), we acknowledge that 'time' and 'space' both operate in close partnership in the global team context. How they serve to structure team dynamics and facilitate distributed team outcomes are topics about which we still have much to learn. It is arguable whether a research programme could productively examine the one without the other. Nonetheless, although 'space' itself is also multifaceted and has local and global dimensions, it could itself be the subject of an independent study, in contrast to a fuller investigation where it is linked inherently to time.

A further and longer term critical concern, we believe, is what impact might sensitively managing these conflicting types of time in a distributed setting have on the sustainability of global software development? This could be a highly fruitful research agenda to move global software development in its worst instantiations from a highly unstable and primitive form of "global labor arbitrage" [33, 34, 35], to a constructive and effective way of sharing expertise and creating software systems in global partnerships based upon mutual respect.

\section{CONCLUSION}

We have outlined the operation of time in a globally distributed team context, based upon an in-depth empirical study into technology-use mediation in global virtual teams. Through the grounded analysis of a rich body of data we have been able to lend credence, with evidence from a global team setting, to prior work that has promoted the multidimensional nature of time. At the very least this suggests that the 'follow the sun' type model accounts for only some of the influence time can have on a global undertaking. Appropriate for an exploratory study such as this, the analysis has informed the identification of a set of questions and strategies that a manager should address when considering the work of a global team. It has also rather naturally led to us uncovering many more research questions and opportunities relevant to the complex phenomenon of time in this context. We conclude that yes, time may well be money, but in the workings of a global team it is much more than that. Given the cultural, social, personal, professional and political dimensions that are associated with the operation of time in this context a much broader characterization of the phenomenon is needed if the actions and interactions of teams and team members are to be leveraged - and supported - most effectively. 


\section{ACKNOWLEDGMENT}

We wish to thank our colleagues and students at AUT University, Uppsala and St Louis Universities, for their cooperation and support in making this research possible.

\section{REFERENCES}

[1] J. Treinen and S. Miller-Frost, "Following the sun: Case studies in global software development," IBM Systems Journal, vol. 45, pp. 773-783, 2006.

[2] A. Gupta and S. Seshasai, "24 Hour Knowledge Factory: Using Internet Technology to Leverage Spatial and Temporal Separations," ACM Transactions on Internet Technology, vol. 7, pp. 14-22, 2007.

\section{[3] S. Seshasai and A. Gupta, "The Role of Information}

Resources in Enabling the 24 Hour Knowledge Factory, "Information Resource Management Journal, vol. 20, pp. 105-127, 2007.

[4] J. McGrath and J. Kelly, Time and Human Interaction: Toward a Social Psychology of Time New York: The Guilford Press, 1986.

[5] A. Powell, G. Piccoli, and B. Ives, "Virtual Teams: A Review of Current Literature and Directions for Future Research," The DATABASE for Advances in Information Systems, vol. 35, pp. 6-36, 2004.

[6] S. Sarker and S. Sahay, "Implications of space and time fordistributed work: an interpretive study of USNorwegian systems development teams," European Journal of Information Systems, vol. 13, pp. 3-20, 2004.

[7] J. Nandhakumar, "Managing Time in a Software Factory: Temporal and Spatial Organization of IS Development Activities," The Information Society, vol. 18, pp. 252-262, 2002.

[8] J. George and L. Jessup, "Groups Over Time: What are WeReally Studying?," International Journal of Human- Computer Studies, vol. 47, pp. 497 -511, 1997.

[9] B. Mennecke and J. Hoffer, "The Implications of GroupDevelopment and History for Group Support System Theory and Practice," Small Group Research, vol. 23, pp. 524-572, 1992.

[10] H. Arrow, M. Poole, K. Henry, S. Wheelan, and R. Moreland, "Time Change and Development -The Temporal Perspective on Groups," Small Group Research, vol. 35, pp. 73-105, 2004.

[11] J. McGrath, "Time Interaction and Performance (TIP): atheory of groups," Small Group Research, vol. 22, pp. $147174,1991$.

[12] H. Arrow, K. Henry, M. S. Poole, S. Wheelan, and R. Moreland, "Traces, Trajectories and Timing -The Temporal Perspective On Groups," in Theories of Small Groups - Interdisciplinary Perspectives, M. S. Poole and A. Hollingshead, Eds. Thousand Oaks: SAGE, 2005, pp. 313-367.
[13] C. Gersick, "Time and transition in work teams: Toward a new model of group development.," Academy of Management Journal, vol. 31, pp. 9-41, 1988.

[14] A. Massey, M. Montoya-Weiss, and Y. Hung, "Because Time Matters: Temporal Coordination in Global Virtual Project Teams," Journal of Management Information Systems, vol. 19, pp. 129-155, 2003.

[15] M. Maznevski and K. Chudoba, "Bridging Space OverTime: Global Virtual Team Dynamics and Effectiveness," Organization Science, vol. 11, pp. 473492, 2000.

[16] J. Walther, "Time Effects in Computer-Mediated Groups: Past, Present and Future," in Distributed Work, P. Hinds, S. Kiesler, and J. Valacich, Eds. Cambridge: The MIT Press, 2002, pp. 235-257.

[17] C. Saunders, C. Van_Slyke, and D. Vogel, "My time or yours? Managing time visions in global virtual teams," Academy of Management Executive, vol. 18, pp. 19-31, 2004.

[18] T. Mitchell and L. James, "Building Better Theory: Timeand the Specification of When Things Happen," Academy of Management Review, vol. 26, pp. 530 -547, 2001.

[19] C. Saunders, "Editor's Comments - Perspectives on Time," MIS Quarterly, vol. 31, pp. iii-xi, 2007.

[20] C. Hampden-Turner and F. Trompenaars, Building Cross Cultural Competence: How to Create Wealth from Conflicting Values. New Haven, Connecticut: Yale University Press, 2000.

[21] G. Hofstede, Culture's Consequences: International Differences in Work-Related Values. Beverley Hills, California: SAGE, 1980.

[22] W. Orlikowski and J. Yates, "It's About Time: TemporalStructuring in Organizations," Organization Science, vol. 13, pp. 684-700, 2002.

[23] G. Klyne and P. Newman, "RFC 3339: Date and Time on the Internet: Timestamps," The Internet Society, Request for Comments Jul 2002.

[24] A. Massey and M. Montoya-Weiss, "Unravelling the Temporal Fabric of Knowledge Conversion: A Model ofMedia Selection and Use," MIS Quarterly, vol. 30, pp. 99 114, 2006.

[25] B. Harmer and D. Pauleen, "Time and Tide," University of Auckland Business Review, vol. 10, pp. 2128, 2008.

[26] Orlikowski, W., Yates, J., Okamura, K. and Fujimoto, M. Shaping Electronic Communication: The Metastructuringof Technology in the Context of Use. Organization Science, 6 (4) 1995, 423 - 444

[27] T. Clear, "Supporting the Work of Global Virtual Teams: The Role of Technology-Use Mediation," in Computing and Mathematical Sciences Auckland: Auckland University of Technology, 2008, (Unpublished) pp. 1-473, http://hdl.handle.net/10292/650.

[28] DSDM, "Guidelines for applying DSDM in an OffshoreEnvironment," DSDM Consortium. Retrieved 

2005.

[29] A. Lee, "Electronic Mail as a Medium for Rich Communication: An Empirical Investigation using Hermeneutic Interpretation," MIS Quarterly, vol. 18, pp. 143-158, June 1994.

[30] Glaser, B. and Strauss, A. The Discovery of Grounded Theory. Sociology Press, Mill Valley, CA, 1967.

[31] Pozzebon, M. and Pinsonneault, A. Challenges in Conducting Empirical Work Using Structuration Theory: Learning from IT Research. Organization Studies, 26 (9) 2005, 1353-1376.

[32] K. Mansfield, "At the Bay," in The Garden Party Katherine Mansfield's New Zealand Stories Illustrated ed: Century Hutchinson, 1987, pp. 85-116.

[33] J. Brecher, T. Costello, and B. Smith, "International Labor Solidarity: The New Frontier," New Labor Forum, vol. 15, pp. 9-18, 2006.

[34] E. Carmel and P. Tjia, Offshoring Information Technology: Sourcing and Outsourcing to a Global Workforce. Cambridge, UK Cambridge University Press, 2005.

[35] V. Casey, "Imparting The Importance Of Culture To GSD In the University Environment," ACM Inroads, 2010 (in press). 